\title{
ENCONTRO DO PLASMODIUM(S) TROPIDURI NO ESTADO DE SÃO PAULO, BRASIL
}

Eduardo Olavo da ROCHA E SILVA* Décio de Camargo RODRIGUES **

$\begin{array}{r}\text { RSPU-B/213 } \\ \hline\end{array}$

Rocha E Silva, E. O. da \& Rodrigues, D. de C. - Encontro do Plasmodium (S) tropiduri no Estado de São Paulo, Brasil. Rev. Saúde públ., S. Paulo, 8:163-70, 1974 .

Resumo: Relata-se o encontro, pela primeira vez no Estado de São Paulo - Brasil, de lagartos do gênero Tropidurus, parasitados pelo Plasmodium (S) tropiduri, mencionando alguns dos achados anteriores do parasito em outras áreas do pais e na Venezuela. Säo salientadas as baixas parasitemias encontradas e a semelhança morfológica das formas estudadas com a descrição do parasita feita por Aragão e Neiva. Säo mostradas ainda as dificuldades no achado das formas exoeritrociticas encontradas no trombócitos. Assinala-se o encontro em baixa densidade, do A. (N) evansae e A. (N.) argyritarsis, numa das äreas estudadas. pidurus.

Unitermos: Plasmođium tropiduri*; Malária*; Säo Paulo, Brasil*; Tro-

\section{I N T RODUCAO}

Recentemente foi relatado em nota preliminar, o encontro de uma hemogregarina hepatozoica no tubo intestinal do Triatoma arthurneivai Lent e Martins, 1940, capturados entre pedras (granito róseo), vivendo em perfeita associação alimentar com lacertídeos (Tropidurus torquatus Wied.) também portadores do hemosporídeo (Rocha E Silva \& PattoLI ${ }^{8}$, 1971).

$\mathrm{Na}$ tentativa do encontro de outros fo- cos da hemogregarina, deparamos em três localidades, com exemplares de Tropidurus torquatus infetados por hematozoários da família Plasmodidae que acreditamos serem os primeiros achados, no Estado de São Paulo, do Plasmodium tropiduri Aragão e Neiva, 1909. Posteriormente, Garnham fazendo uma revisão dos plasmódios de lacertídeos, o colocou no subgênero Sauramoeba. Este plasmódio, foi descrito por Aragão e Neiva em 1909,

* Da Superintendência do Saneamento Ambiental (SUSAM) da Secretaria da Saúde do Estado de São Paulo - Rua Tamandaré. 649 - São Paulo, SP - Brasil. Do Departamento de Epidemiologia da Faculdade de Saúde Pública da USP - Av. Dr. Arnaldo, 715 São Paulo, SP - Brasil.

* Do Departamento de Parasitologia do Instituto de Clências Biomédicas da USP - Cidade Universitária - São Paulo, SP - Brasil. 
ROCHA E SILVA, E. O. da \& RODRIGUES, D. de C. - Encontro do Plasmodum (S) tropiaum no Estado de São Paulo, Brasil. Rev. Saúde públ., S. Paulo, 8:163-70, 1974.

ao examinar o sangue de Tropidurus torquatus enviados por Carlos Chagas, procedentes de Bicudos (Minas Gerais).

L'm novo encontro do parasita, somente veio a ocorrer, quando CARINI ${ }^{2,3}$ 11941) consignou sua presença no sangue de um lagarto, inicialmente classificado no gênero Stenocercus, mas em seguida corrigido para Tropidurus, capturado em Porto Nacional, Estado de Goiás. A seguir, Pessol \& Lopes ${ }^{\top}$ (1963), examinando 39 exemplares de Tropidurus torquatus procedentes de Jacobina, na Bahia. assinalaram a possível presença do $P$. (S.) tropidurus, no sangue de quatro exemplares. No Estado do Amazonas, região de Codajaz, $\mathbb{W}^{\top}{ }_{\text {alliKer }}{ }^{11}$ (1963), em esfregaços de sangue de três Ameiva ameiva, encontrou um plasmódio morfologicamente parecido com o P.(S.) tropiduri, aventando no entanto, a hipótese de que poderia se tratar de espécie nova. Também na Amazônia (Pará), Lainsox \& SHAW $^{\circ}$ (1969), em 20 exemplares de Mabuya mabouya examinados, encontraram 8 infetados por este plasmódio, sendo que seis deles albergavam ainda o $P$. dipoglossi.

Fora do Brasil, conseguimos obter referência da sua presença, apenas na Venezuela, onde Scorza ${ }^{3.11}$ (1971), ao apresentar os resultados do estudo das formas sanguícolas do parasita à luz da microscopia eletrônica, relatou o encontro no país, de exemplares do Tropidurus torquatus naturalmente infetados pelo P.(S.) tropiduri. No seu trabalho, realça a profunda semelhança morfológica entre suas formas $\mathrm{e}$ as formas sanguícolas do $P$. (S) floridense.

\section{MATERIAI E MÉTODOS}

\subsection{Pracedência}

Os exemplares de Tropidurus torquatus (Figura 1) que tivemos oportunidade de encontrar, parasitados pelo Plasmo- dium (S.) tropiduri, foram capturados entre 1971 e 1973, em três municípios do Estado de São Paulo (Figura 2), a saber:

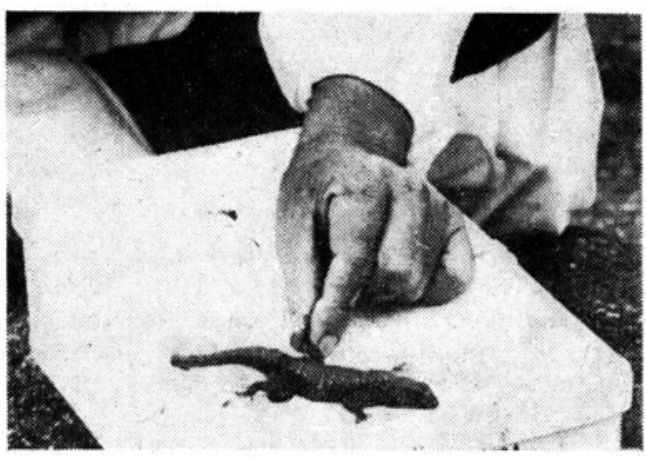

Fig. 1 - Exemplar do Tropudurus torquatus Wied.

a) Município de Divinolândia - Bairro Cachoeirinha

Do total de 51 lagartos capturados. em quatro pesquisas de campo realizadas, dez exemplares se apresentaram infetados pelo $P$. (S.) tropiduri, equivalendo a um indice de infecção de $19,6 \%$.

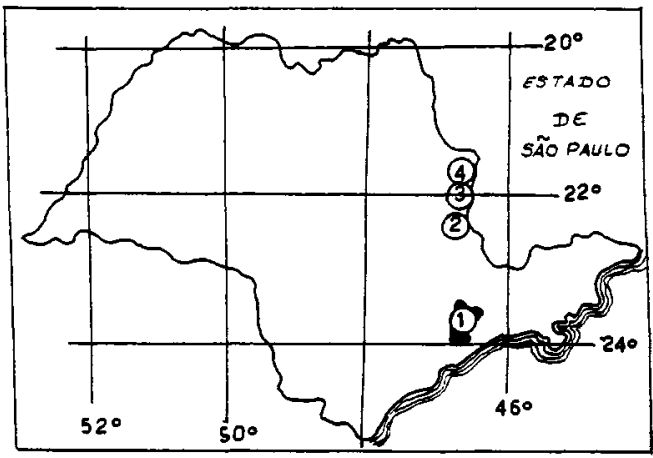

Fig. 2 - Mapa

$$
\begin{aligned}
& 1 \text { - São Paulo (capital) } \\
& 2 \text { - Município de Pinhal } \\
& 3 \text { - Município de Aguas da Prata } \\
& 4 \text { - Municipio de Divinolândia }
\end{aligned}
$$


ROCHA E SILVA, E. O. da \& RODRIGLES, D. de C. - Encontro do Plasmodum (S) tropiluri no Esiado de São Paulo, Brasil. Rev. Saúde públ., S Paulo, 8:163-70, 1974.

Nessa localidade, foram realizadas algumas capturas entomológicas que visaram a obtenção de informações sobre os possíveis vetores na área. Essas capturas revelaram, talvez em virtude da época em que foram realizadas, a presença de um número muito pequeno dos seguintes anofelinos:

Anopheles (Nyssorhynchus) evansae (Brethes, 1926) e Anopheles (Nyssorhynchus) argyritarsis Robineau-Desvoidy, 1827.

Os exames das glândulas salivares dos poucos exemplares capturados, revelaramse negativos para esporozoitas e o exame das paredes do estômago de um exemplar de Anopheles (N.) evansae, mostrou a presença de formas semelhantes a oocistos. b) Municipio de Pinhal

\section{- Bairro Boa Vista}

Em três pesquisas de campo realizadas, foram capturados 31 lagartos, tendo sido encontrados infetados 2 exemplares, proporcionando o índice de infecção de $6,4 \%$.

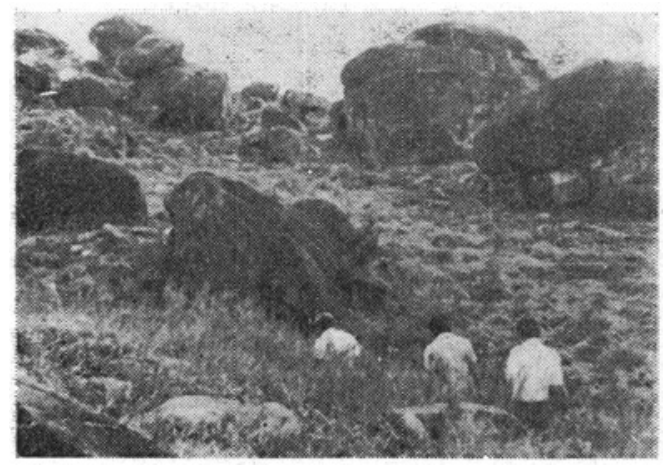

Fig. 3 - Vista parcial do Bairro Boa Vista, mostrando as pedras onde vivem os lacertideos.

Neste bairro, não foram realizadas pesquisas entomológicas.

$\mathrm{Na}$ Figura 3, damos uma visão parcial do Bairro Boa Vista, focalizando o "habitat" do Tropidurus torquatus.
Na Figura 4, um detalhe dos ecótopos deste lacertídeo, salientando que são encontrados nas fendas e espaços entre pedras justapostas e, algumas vezes, capturados juntamente com pequenos roedores, quirópteros e artrópodes diversos.

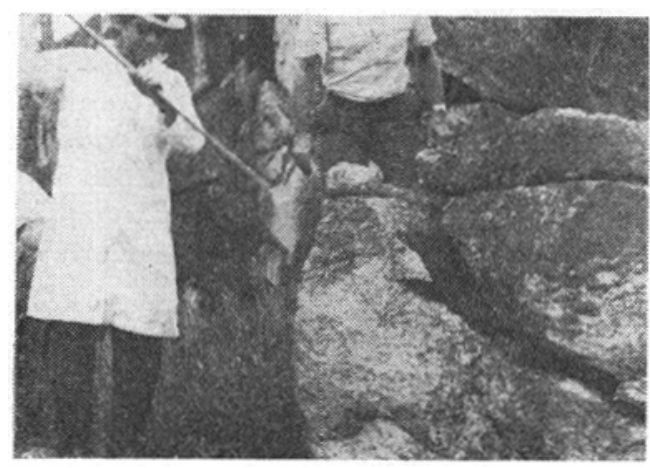

Fig. 4 - Detalhes de pedras e fendas, onde são encontrados exemplares do Tropidurus torquatus.

c) Municipio de Aguas da Prata - Bairro Fonte Platina

As cinco visitas realizadas à localidade em busca de lacertídeos, possibilitaram a captura de 49 exemplares de Tropidurus torquatus, dos quais 6 infetados pelo $P$. (S.) tropiduri, ou seja, $12.2 \%$ dos capturados.

Nesta localidade, vale a pena frisar, constatou-se que além da presença do hematozoário ora em pauta, estudos em andamento nos levam a considerar como provável, a presença de outra espécie de plasmódio parasitando alguns dos lacertídeos capturados.

As características dos ecótopos do Tropidurus torquatus nas três áreas estudadas, não diferem em seus aspectos gerais, da descrição dos mesmos feita por FoRattini et al. ${ }^{1}$ (1968). 
ROCHA E SILVA, E. O. da \& RODRIGUES, D. de C. - Encontro do Plasmodium (S) tropiduri no Estado de São Paulo, Brasil. Rev. Saúde públ., S. Paulo, 8:163-70, 1974.

\subsection{Estudo das formas do plasmódio:}

\section{a) Esquizogônia eritrocitica}

O estudo das formas eritrocíticas do Plasmodium (S.) tropiduri foi realizado em esfregaços de sangue de lagartos, colhidos por punção direta do coração $e$ corados pelo método de Giemsa.

A parasitemia natural quase sempre se revelou baixa. Poucos lagartos apresentaram parasitemia elevada, permitindo o estudo das várias formas evolutivas do parasita e sugerindo a inexistência de sincronismo no desenvolvimento das formas assexuadas.

Os parasitas se localizam numa das extremidades das hemácias e mesmo as formas maiores, como os gametócitos que são arredondados, parecem não interferir na posição do núcleo e forma das hemácias parasitadas. $\mathrm{O}$ mesmo não ocorre quando o parasitismo se processa nos eritrócitos jovens.

Os trofozoitas jovens do Plasmodium (S.) tropiduri, em pequeno número nos esfregaços estudados, apresentam em geral, formas arredondadas, citoplasma corado em azul, com ou sem vacúolo e pequeno fragmento (vermelho) de cromatina. A maioria das formas observadas, representada por trofozoitas médios (Fi-

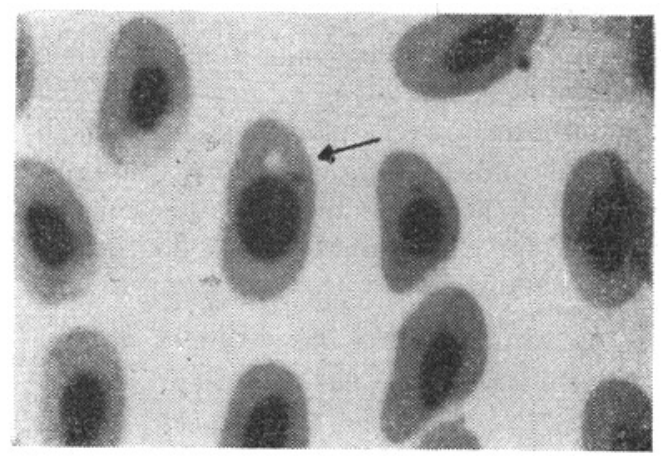

Fig. 5 - Trofozolta médio. gura 5), bem como, esquizontes e gametócitos, são encontrados em diferentes fases de evolução.

Nas formas mais desenvolvidas de esquizonte (Figura 6), nota-se a presença de pigmento, sob a forma de granulações finas com tendência a se acumular em pontos do citoplasma. Estas formas, apresentam contornos com discretas irregularidades, sugerindo pequena mobilidade do. parasita no interior das hemácias.

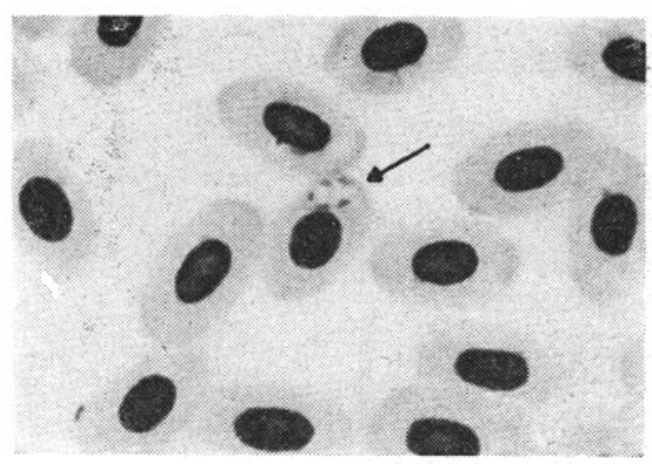

Fig. 6 - Erquizonte médio.

Os esquizontes maduros, geralmente produzem oito merozoitas, raramente dez a doze, aglomerados, dispostos em círculo ou em leque, apresentando os grânulos de pigmento reunidos em um ponto (Figura 7).

Os microgametócitos (Figura 8) apresentam a cromatina frouxa, fracamente corada em róseo, distribuida pela área central do citoplasma. Este, debilmente corado em azul ou róseo, apresenta as gcanulações do pigmento geralmente finas e irregularmente dispostas. Os macrogametócitos por sua vez, apresentam o citoplasma denso, corado em azul, com a cromatina nas proximidades das margens. Os grãos de pigmento se concentram junto à cromatina ou em determinado ponto do citoplasma. 
ROCHA E SILVA, E. O. da \& RODRIGUES, D. de C. - Encontro do Plasmodium (S) tropiduri no Estado de São Paulo, Brasil. Rev. Saúde públ., S. Paulo, 8:163-70, 1974.

Os aspectos morfológicos relatados acima não diferem. em linhas gerais, da descrição do parasita feita inicialmente por Aragão e Neiva e posteriormente, por Garnham. No entanto, parece-nos de interesse salientar as principais diferenças existentes entre o plasmódio em estudo e os demais que se agrupam, segundo Garnham, no subgênero Sauramoeba. Notadamente as espécies encontradas nas Américas que apresentam também gametócitos arredondados: $P$. (S.) mexicanum e P. (S.) floridense.

As diferenças em relação ao $P$. (S.) mexicanum prendem-se, entre ouiras, aos gametócitos que nesta são de grande tamanho, deformando de maneira acentuada as hemácias, o que não ocorre no $P$. (S.) tropiduri. As diferenças em relação ao $P$. (S.) fioridense são menos evidentes e se prendem, sobretudo, à indiferente posição ocupada por este no eritrócito, bem como, a existência nesta espécie de esquizontes maduros com mais de doze merozoitas.

No que se refere aos demais plasmódios do subgênero, como o P. (S.) dipoglossi e $P$. (S.) cnemidophori, a forma alongada dos gametócitos é um dos elementos básicos de distinção.

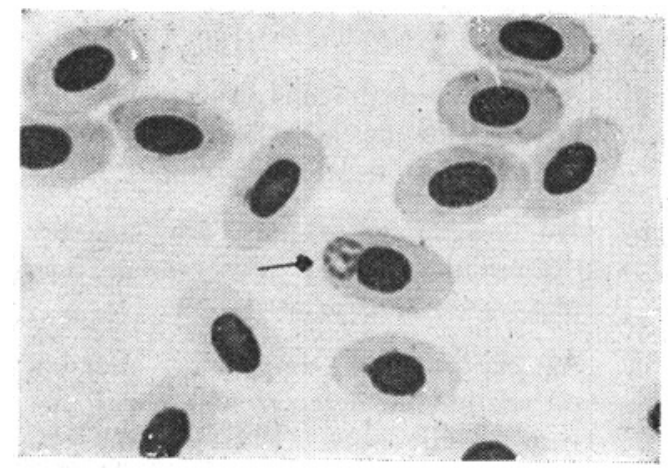

Fig. 7 - Erquizonte maduro.

\section{b) Esquizogônia exoeritrocitica}

Scorza ${ }^{9}$ (1971), examinando 54 lacertídeos identificados como sendo o Tropidurus torquatus, faz referência ao encontro em 4 lagartos, de formas exoeritrocíticas do Plasmodium tropiduri no interior de trombócitos, inclusive refere a presença de formas assexuadas e sexuadas.

Nós, também, tivemos oportunidade de encontrar em esfregaços de sangue, de dois Tropidurus torquatus, naturalmente infetados pelo Plasmodium (S.) tropiduri. formas correspondentes a esquizontes exoe-

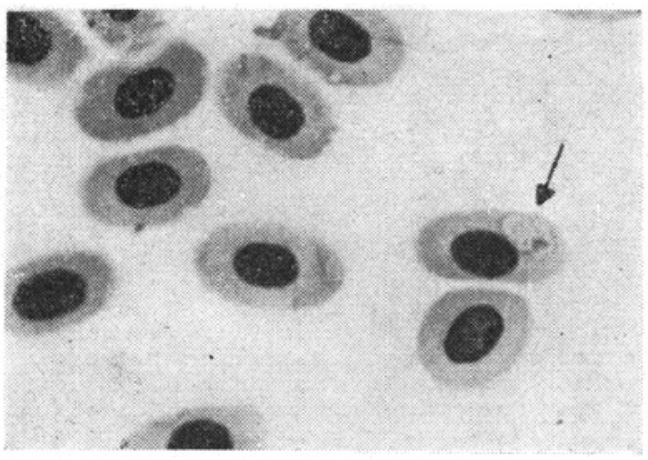

Fig. 8 - Forma sexuada (Microgametócito).

ritrocíticos. Por outro lado, em lacertídeos infetados experimentalmente através de inoculações intratorácicas, tivemos oportunidade de observar algumas formas exoeritrocíticas em trombocitos (Figura 9), em diferentes fases de sua evolução. $\mathrm{Na}$ Figura 10 apresentamos outra das formas vistas, esta com mais de vinte fragmentos de cromatina, no caso, um esquizonte maduro apigmentado. As formas apigmentadas em trombócitos, ocorreram, concomitantemente com a presença de abundantes formas eritrocíticas pigmentadas.

Essas observações, coincidem em parte com as relatadas por Scorza ${ }^{10}$ (1971), 
ROCHA E SILVA, E. O. da \& RODRIGUES, D. de C. - Encontro do Plasmodium (S) tropiduri no Estado de São Paulo, Brasll. Rev. Saúde públ., S. Paulo, 8:163-70, 1974.

uma vez que constatamos também a presença de formas pigmentadas do Plasmodium (S.) tropiduri nos trombócitos, porém, em nenhuma oportunidade nos foi possivel ver com nitidez a membrana pericelular deste elemento figurado do sangue dos lagartos, cujo papel é participar da coagulação sanguínea.

Ainda na tentativa de detectar formas exoeritrocíticas tissulares do Plasmodium (S.) tropiduri, realizamos vários cortes de tecidos: coração, vasos, pulmões, cérebro, fígado e médula óssea de lagartos infetados. Estes cortes ou macerados, co-

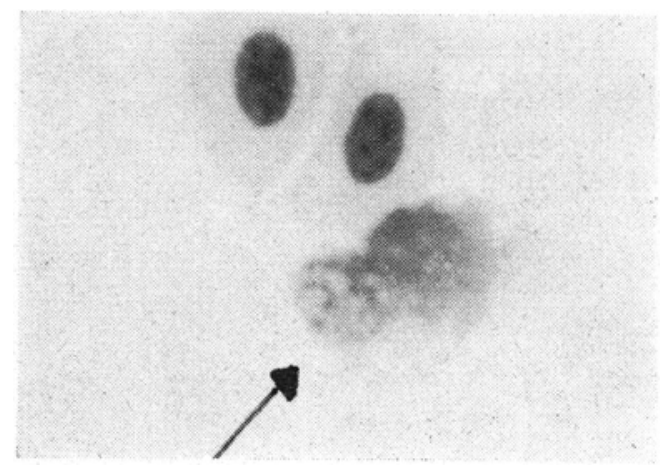

Fig. 9 - Forma exoeritrocitica em trombócito.

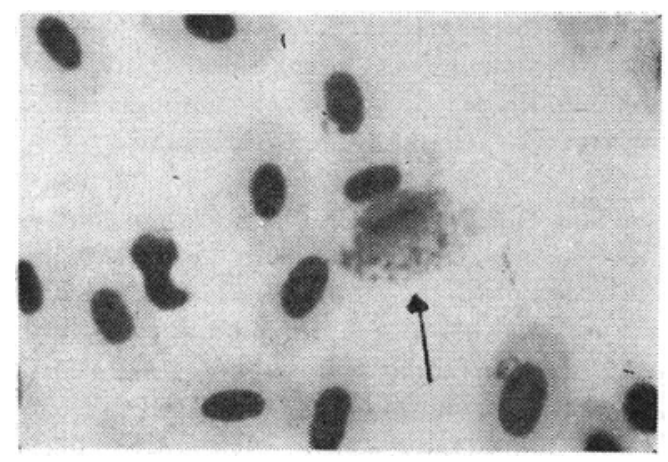

Fig. 10 - Forma exoeritrocitica (merozoitas visiveis) em trombocito. rados respectivamente pela hematoxilina-eosina e Giemsa, sempre se mostraram negativos.

\section{c) Infecções experimentais}

As tentativas de manutenção em laboratório do Plasmodium (S.) tropiduri, através de inoculações, foram bem sucedidas, uma vez que os exames de sangue dos lagartos inoculados revelaram a presença, em alta densidade, de formas assexuadas e sexuadas do plasmódio. Embora não se afirme categoricamente que se trata de nova infecção, desde que os lagartos inoculados não foram criados em laboratório, isso provavelmente deve ter ocorrido algumas vezes. Por outro lado, a simples exacerbação (recaída) de antiga infecção, embora pouco provável nada tem de absurdo, tendo em vista observações de que a infecção em pauta, nas áreas trabalhadas, parece ter prevalência maior do que a registrada pela simples coleta de uma ou duas amostras de sangue.

No que se refere as relações parasitahospedeiro, mantidas no caso, entre plasmódio e lacertídeo, como era de se esperar, não aparentam maior agressividade, tendo em vista a ausência de modificações flagrantes de aspecto e comportamento, comparados lagartos infetados $\mathrm{e}$ não infetados.

\section{CON CLUSOE S}

a) A procura de formas exoeritrocíticas, nos tecidos de lagartos naturalmente infetados, se mostrou negativa.

b) No sangue de alguns dos lagartos examinados, foram observadas formas exoeritrocitárias.

c) As capturas de dípteros, realizadas no sentido de detectar os vetores, foram insuficientes. Entre outros fatores, devido à baixa densidade dos alados presentes na ocasião em que 
ROCHA E SILVA, E. O. da \& RODRIGLES, D. de C. - Encontro do Plasmodium (S) tropidur no Estado de São Paulo, Brasil. Rev. Saúde públ., S. Paulo, 8:163-70, 1974.

foram realizadas. No entanto, em uma das localidades, foi assinalada a presença do Anopheles (N.) evansae e Anopheles (N.) argyritarsis.

d) Através de inoculações em laboratório, a infecção aparentemente foi transferida para lacertídeos que inicialmente apresentavam exames de sangue negativos.

\section{A GRADECIMENTOS}

À Diretoria de Combate a Vetores da SUSAM, pelas facilidades proporcionadas e aos servidores (auxiliares-técnicos\}. Srs. José de Campos Camargo, Antenor do Nascimento Ferraz Filho e Álvaro de Oliveira Santos, pela colaboração prestada.

Rocha E Silva, E. O. da \& Rodrigues, D. de C. - [Find of Plasmodium (S) tropiduri in the State of S. Paulo, Brazil.] Rev. Saúde públ., S. Paulo, 8:163-70, 1974

Summary: The find, for the first time in the state of sano Paulo Brasil, a parasite, the Plasmodium (S.) tropiduri in lizards of genus Tropidurus is described. Initially it was related this parasite found, by other authors, in another areas of the Brasil and Venezuela. As far as the parasitology itself is concerned, the low parasite density encountered and the simillar morfology as it was studied by Aragão et Neiva are reported. There remain the difficulties encountered in the discovery of exoeritrocitic forms in trombocytes. In entomological research, finding in low density, of the A. (N.) evansae and the A. (N.) argyritarsis is reported.

Uniterms: Plasmodium tropiduri*; Malaria*; S. Paulo (Brazll)*: Tropidurus.

\section{REFERENCIAS BIBLIOGRAFICAS}

1. ARAGAO, H. de B. \& NEIVA, A. - Contribuição para o estudo dos parasitas intraglobulares dos lacertidas. Mem. Inst. Oswaldo Cruz, 1:44-50, 1909.

2. CARINI, A. - Sobre um plasmodium endoglobular de um lagarto. Arq. Biol., S. Paulo, 25:46-7, 1941.

3. CARINI, A. - Retificações necessárias. Arq. Biol, S. Paulo, 24:121-2, 1941

4. FORATTINI, O. P. et al. - Dados sobre a biologia do Triatoma arthurneivai no sudeste do Estado de São Paulo, Brasil (Hemiptera, Reduvidae). Rev. Saúde puibl., S. Paulo, 2:186-93, 1968.
5. GARNHAM, P. C. C. - Malaria Parasites and other Haemosporidia. Oxford, Blackweel Scientific Publication, 1966.

6. IAINSON, R, \& SHAW, J. J. - New host records for Plasmodium dipoglossi, $P$. tropiduri Aragão and Nelva, 1909, and $P$. cnemidophori Car1ni, 1941. Parasitology, 59:163-70. 1969.

7. PESSOA, S. B. \& LOPES, J. A. S. Nota sobre alguns hematozoárıos de lagartixas de Jacobina (Bahia, Brasil). Rev. Inst. Med. trop. São Palclo, $5: 133-9,1963$. 
ROCHA E SILVA, E. O. da \& RODRIGUES, D. de C. - Encontro do Plasmodium (S) tropiduri no Estado de São Paulo, Brasil. Rev Saúde públ., S. Paulo, 8:163-70, 1974.

8. ROCHA e SILVA, E. O, da \& PATTOLI, D. B. G. - Considerações sobre a presença de uma Hemogregarina no tubo digesitvo de exemplares do Triatoma arthurneivai. Rev. paul. Med., 78:91, 1971.

9. SCORZA, J. V. - Electron microscope study of the stages of Plasmodium tropiduri, a lizard malaria parasite. Parasitology, 63:1-20, 1971.

10. SCORzA, J. V. - Assexual and sexual stages of a malaria parasite in the trombocytes of Tropidurus torquatus (Iguantdae) infected with Plasmodium tropiduri. J. Protozoology, 18: 403-10, 1971.

11. WALLIKER, D. - Malaria parasites of some Brasilian lizards. Parasitology, 56:39-44, 1946 .

Recebıdo para publicaçāo em 19/2/1974 Aprovado para publicasão em 24/4/1974 